\title{
EDITORIAL
}

\section{Moving albumin into the small volume resuscitation era}

\author{
Fernando G. Zampieri ${ }^{1,2^{*}}$ and Peter Buhl Hjortrup ${ }^{3}$
}

(0) 2018 Springer-Verlag GmbH Germany, part of Springer Nature and ESICM

Physicians have an intense 70-year history of enthusiasm, skepticism, fear, and reconciliation with albumin products since their market introduction in the late 1940s [1]. Despite its cumbersome production method and costs, albumin became popular soon after its debut. Advances in its production technique, producing purer formulations with less prekallikrein activators, turned it into a compound with few immediate adverse reactions that appeared to be safe [1]. However, the most recent turbulent episodes of albumin use (still known by most critical care physicians) start with the notorious meta-analysis suggesting harm [2], passes through a redemption clinical trial [3], and lands on more recent studies that may point to a specific direction, but yet provide no conclusive data [4]. There are still several gaps in our knowledge about albumin resuscitation. One of the most pressing questions is whether, given that higher positive fluid balance is associated with worse clinical outcome [5] and that albu$\mathrm{min}$ is safe [3], a resuscitation strategy based on hyperoncotic (20\%) albumin resuscitation would be useful? If so, which would be the ideal comparator: isoncotic albumin or crystalloids? While comparing $20 \%$ albumin to crystalloids would make sense considering the cost of high albumin, one would be left unsure if differences would be due to the presence of albumin by itself or, maybe, due to the side effects of some crystalloids solutions (especially

*Correspondence: fgzampieri@gmail.com

1 Research Institute, HCor-Hospital do Coração, São Paulo, Brazil

Full author information is available at the end of the article saline), so isoncotic albumin seemed like a rational comparator for a first peek. The first step to answer this question is now discussed in the paper by Mårtensson and coworkers (the SWIPE trial) in a recent article in Intensive Care Medicine [6]. In this well-conducted pilot study, 330 patients (out of an expected 400 patients) from Australia and UK were randomized to receive $20 \%$ or $4-5 \%$ albumin preparations in the first $48 \mathrm{~h}$ after ICU admission. As a result of the study's pilot nature, the authors were interested in physiological effects and (appropriately) chose cumulative volume of resuscitation fluid as their primary outcome. Rich data on physiological and hemodynamic variables also allow us to obtain a good picture of how the interventions differed.

Close inspection of the randomized population shows that most (close to $70 \%$ ) patients were admitted after surgery (mostly elective procedures). The baseline values of arterial pressure and the relatively low number of patients receiving norepinephrine corroborate the idea that patients were not severely hypotensive at randomization. Finally, volume of resuscitation fluid was low in both arms (even lower in $20 \%$ albumin groups, as we will discuss later). All these findings suggest that the trial included a low-risk population. Consequently, rates of acute kidney injury and hospital mortality were low.

However, important information can be obtained from the trial. Patients in the $20 \%$ group indeed received less fluid ( $450 \mathrm{~mL}$ less), had their albumin levels increased by $3 \mathrm{~g} / \mathrm{L}$ more while having similar urinary output. This is an important finding, since increase in plasma oncotic pressure could theoretically decrease glomerular filtration rate and reduce diuresis [7], which could minimize the impact of a lower fluid infusion volume on total fluid balance as a result of a reduction in urinary output. The net effect seen in SWIPE was a lower fluid balance in the $20 \%$ group (difference of approximately $570 \mathrm{~mL}$ ). Hemodynamic trends were mostly similar. Changes in

\section{Giti Springer}




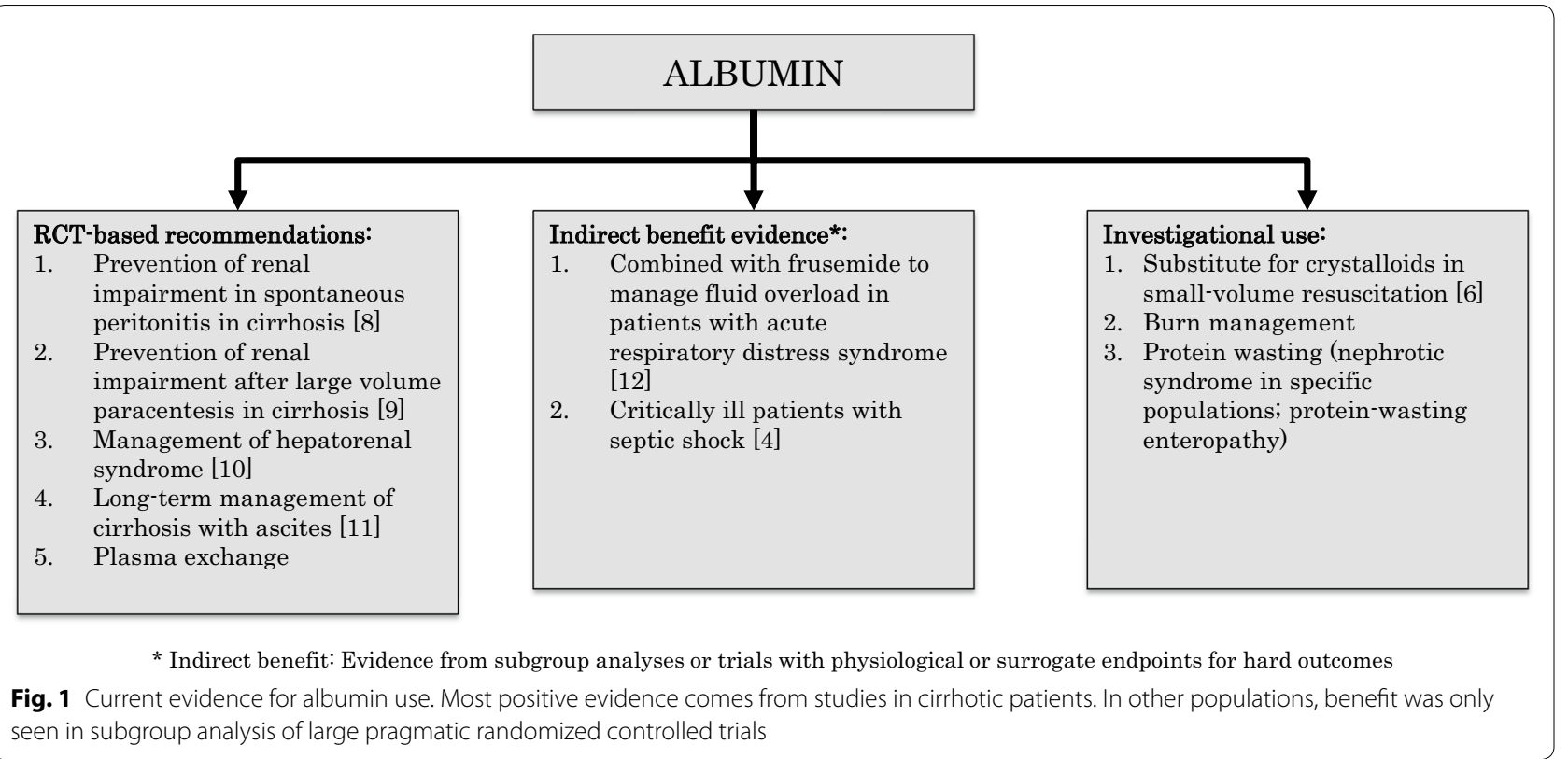

electrolyte levels were small, albeit statistically significant, with unclear clinical significance. Lack of blinding could, as always, have played a role in many of the outcomes assessed by the authors, especially total infused volume. Physicians could, for example, consider that true fluid expansion with $20 \%$ albumin would be twice the one expected with $4-5 \%$ albumin and therefore interrupt earlier fluid resuscitation. From the SAFE trial, however, we know that in practice, when blinding is applied, albumin $4 \%$ use results in a more modest fluid-sparing effect when compared to crystalloids (1:1.3). Would the same happen if blinding was applied when comparing $20 \%$ to $4-5 \%$ albumin?

Secondary results of SWIPE show no clear signal of harm associated with $20 \%$ albumin (similar occurrence of acute kidney injury, mortality, and survival). These findings should be interpreted with caution, since the low number of patients and the low illness severity constrain both the power to detect difference and generalization of the results. Although the authors report that no heterogeneity in treatment effect was seen between important subgroups (including septic patients), all subgroups were small, which hampers any reliable conclusion about this finding.

The SWIPE trial was a necessary step prior to a large randomized controlled trial (RCT) comparing different albumin concentrations in critically ill patients. We are left wondering whether future large-scale RCTs will show an overall benefit of $20 \%$ albumin vs. $4 / 5 \%$ albumin (or crystalloids). If so, would it be related to lower cumulative fluid balance? Would it be mediated by the higher serum albumin levels, which could have anti-inflammatory and adsorptive properties? Maybe lower chloride levels could also play a role? All of the above? These questions highlight how hard and complex any discussion is involving benefits and harms of fluids: there is never one unique mechanism involved. Albumin has a long history of clinical trials, but robust evidence sustaining its use is still scant (Fig. 1). Most high-quality evidence is limited to management of cirrhosis and its complications. We hope that future trials provide more definitive answers in albumin's troubled history.

\section{Author details \\ ${ }^{1}$ Research Institute, HCor-Hospital do Coração, São Paulo, Brazil. ${ }^{2}$ Intensive Care Unit, Hospital Alemão Oswaldo Cruz, São Paulo, Brazil. ${ }^{3}$ Department of Intensive Care, Copenhagen University Hospital, Rigshospitalet, Blegda- msvej 9, 2100 Copenhagen, Denmark.}

\section{Compliance with ethical standards}

Conflicts of interest

Both authors declare they have no conflicts of interest.

Received: 5 July 2018 Accepted: 12 July 2018

Published online: 24 July 2018

\section{References}

1. Matejtschuk P, Dash CH, Gascoigne EW (2000) Production of human albumin solution: a continually developing colloid. Br J Anaesth 85(6):887-895

2. Cochrane Injuries Group Albumin Reviewers (1998) Human albumin administration in critically ill patients: systematic review of randomised controlled trials. BMJ 317(7153):235-240 
3. Finfer S, Bellomo R, Boyce N, French J, Myburgh J, Norton R, SAFE Study Investigators (2004) A comparison of albumin and saline for fluid resuscitation in the intensive care unit. N Engl J Med 350(22):2247-2256

4. Caironi P, Tognoni G, Masson S, Fumagalli R, Pesenti A, Romero M, Fanizza C, Caspani L, Faenza S, Grasselli G, lapichino G, Antonelli M, Parrini V, Fiore G, Latini R, Gattinoni L (2014) Albumin replacement in patients with severe sepsis or septic shock. N Engl J Med 370:1412-1421

5. Boyd JH, Forbes J, Nakada TA, Walley KR, Russell JA (2011) Fluid resuscitation in septic shock: a positive fluid balance and elevated central venous pressure are associated with increased mortality. Crit Care Med 39:259-265

6. Mårtensson J, Bihari S, Bannard-Smith J et al. Small volume resuscitation with 20\% albumin in intensive care: physiological effects. Intensive Care Med. https://doi.org/10.1007/s00134-018-5253-2

7. Legrand $M$, Payen D (2011) Understanding urine output in critically ill patients. Ann Intensive Care 1(1):13
8. Sort P, Navasa M, Arroyo V, Aldeguer X, Planas R, Ruiz-del-Arbol L et al (1999) Effect of intravenous albumin on renal impairment and mortality in patients with cirrhosis and spontaneous bacterial peritonitis. N Engl J Med 341(6):403-409

9. Bernardi M, Caraceni P, Navickis RJ, Wilkes MM (2012) Albumin infusion in patients undergoing large-volume paracentesis: a meta-analysis of randomized trials. Hepatology 55(4):1172-1181

10. Sanyal AJ, Boyer T, Garcia-Tsao G, Regenstein F, Rossaro L, Appenrodt B et al (2008) A randomized, prospective, double-blind, placebo-controlled trial of terlipressin for type 1 hepatorenal syndrome. Gastroenterology 134(5):1360-1368

11. Caraceni P, Riggio O, Angeli P, Alessandria C, ANSWER Study Investigators et al (2018) Long-term albumin administration in decompensated cirrhosis (ANSWER): an open-label randomised trial. Lancet 391(10138):2417-2429 